\title{
BMJ Open Ginkgo biloba extract for prevention of acute mountain sickness: a systematic review and meta-analysis of randomised controlled trials
}

Tou-Yuan Tsai, ${ }^{1,2}$ Shih-Hao Wang, ${ }^{2,3,4}$ Yi-Kung Lee, ${ }^{1,2}$ Yung-Cheng Su ${ }^{1,2}$

To cite: Tsai T-Y, Wang S-H, Lee Y-K, et al. Ginkgo biloba extract for prevention of acute mountain sickness: a systematic review and meta-analysis of randomised controlled trials. BMJ Open 2018;8:e022005. doi:10.1136/ bmjopen-2018-022005

- Prepublication history and additional material for this paper are available online. To view these files, please visit the journal online (http://dx.doi org/10.1136/bmjopen-2018022005).

Received 30 January 2018 Revised 14 June 2018 Accepted 12 July 2018

Check for updates

(C) Author(s) (or their employer(s)) 2018. Re-use permitted under CC BY-NC. No commercial re-use. See rights and permissions. Published by BMJ.

${ }^{1}$ School of Medicine, Tzu Chi University, Hualien, Taiwan

${ }^{2}$ Emergency Department, Dalin Tzu Chi Hospital, Buddhist Tzu Chi Medical Foundation, Chiayi, Taiwan

${ }^{3}$ Department of Physical Medicine and Rehabilitation, Chang Gung Memorial Hospital at Chiayi, Chiayi, Taiwan

${ }^{4}$ Department of Recreation and Leisure Industry Management, College of Management,

National Taiwan Sport University, Taoyuan, Taiwan

Correspondence to

Dr Yung-Cheng Su;

drsu119@gmail.com

\section{ABSTRACT}

Objective Trials of ginkgo biloba extract (GBE) for the prevention of acute mountain sickness (AMS) have been published since 1996. Because of their conflicting results, the efficacy of GBE remains unclear. We performed a systematic review and meta-analysis to assess whether GBE prevents AMS.

Methods The Cochrane Library, EMBASE, Google Scholar and PubMed databases were searched for articles published up to 20 May 2017. Only randomised controlled trials were included. AMS was defined as an Environmental Symptom Questionnaire Acute Mountain Sickness-Cerebral score $\geq 0.7$ or Lake Louise Score $\geq 3$ with headache. The main outcome measure was the relative risk (RR) of AMS in participants receiving GBE for prophylaxis. Metaanalyses were conducted using random-effects models. Sensitivity analyses, subgroup analyses and tests for publication bias were conducted.

Results Seven study groups in six published articles met all eligibility criteria, including the article published by Leadbetter et al, where two randomised controlled trials were conducted. Overall, 451 participants were enrolled. In the primary meta-analysis of all seven study groups, GBE showed trend of AMS prophylaxis, but it is not statistically significant ( $R R=0.68 ; 95 \% \mathrm{Cl} 0.45$ to $1.04 ; \mathrm{p}=0.08$ ). The $\mathrm{I}^{2}$ statistic was $58.7 \%(\mathrm{p}=0.02)$, indicating substantial heterogeneity. The pooled risk difference (RD) revealed a significant risk reduction in participants who use GBE ( $\mathrm{RD}=-25 \%$; $95 \% \mathrm{Cl}$, from a reduction of $45 \%$ to $6 \%$; $p=0.011)$ The results of subgroup analyses of studies with low risk of bias, low starting altitude $(<2500 \mathrm{~m})$, number of treatment days before ascending and dosage of GBE are not statistically significant.

Conclusion The currently available data suggest that although GBE may tend towards AMS prophylaxis, there are not enough data to show the statistically significant effect of GBE on preventing AMS. Further large randomised controlled studies are warranted.

\section{INTRODUCTION}

\section{Background}

Rapid ascent from low to high altitude (>2500 $\mathrm{m}$ above sea level) is often followed by headache, fatigue, shortness of breath, sleeplessness and anorexia, a symptom complex
Strengths and limitations of this study

- This meta-analysis is the first systematic review and meta-analysis evaluating ginkgo biloba extract as an acute mountain sickness prophylactic.

- This meta-analysis was strengthened by a thorough quality assessment of each enrolled study and comprehensive subgroup analyses.

- There is notable heterogeneity and the small number of studies limits the analyses, but heterogeneity decreased after excluding studies with high risk of bias.

- Insufficient power may be an issue in this meta-analysis.

- Further large randomised controlled studies are warranted.

called acute mountain sickness (AMS). ${ }^{1}$ The Lake Louise Score (LLS) questionnaire ${ }^{2}$ and the Environmental Symptom Questionnaire III $^{3}$ are two tools to diagnose and evaluate the severity of AMS. AMS is more likely to happen at altitudes higher than $2500 \mathrm{~m},{ }^{4}$ and worldwide studies reported an incidence of AMS of $25 \%-37 \%$ at $1900-3400 \mathrm{~m}^{15}$ Children are more prone to develop AMS, with an incidence of $59 \%{ }^{6}$

The pathophysiology of AMS is associated with cerebral oedema, with the most compelling evidence coming from the brain MRI study of Hackett et al, ${ }^{7}$ which showed intense T2 signals in the white matter, particularly in the splenium and corpus callosum. Vasogenic leakage increases the permeability of the endothelium, causing an elevation in intravascular pressures and inducing hypoxaemia. In addition, hypoxic ventilatory response and activation of the renin-angiotensin-aldosterone system are also reported to be associated with AMS. ${ }^{8}$ The most effective method to prevent AMS is gradual ascent. The most common pharmacological agent used to prevent AMS is acetazolamide. ${ }^{9}$ However, 
acetazolamide can cause paresthesia, dysgeusia, and sometimes nausea or drowsiness. ${ }^{10}$ Its use is also contraindicated in patients with a history of anaphylaxis to sulfa antibiotics or acetazolamide.

\section{Importance}

Ginkgo biloba extract (GBE) is an option for those seeking a natural alternative treatment. GBE is found to decrease tissue hypoxia, induce vasodilation, and reduce free-radical production and lung leak, which may in turn prevent AMS. ${ }^{11-14}$ Roncin et al ${ }^{5}$ in 1996 published the first study to suggest that GBE can prevent AMS. However, not all subsequent studies have shown benefit. ${ }^{13}{ }^{16-20}$ To date, there is no best evidence to support the effectiveness of GBE.

\section{Goal of this investigation}

The aim of our study was to assess the effectiveness of GBE as prophylaxis for AMS by conducting a meta-analysis and systematic review of the relevant literature.

\section{METHODS}

\section{Databases and search strategy}

We searched the Cochrane Library, EMBASE, Google Scholar and PubMed databases for articles published up to 20 May 2017. No limits were applied to our Boolean search strategy, which included keywords ('Ginkgo', 'Altitude Sickness', 'Mountain'), medical subject headings ('Ginkgo biloba', 'Altitude Sickness') and Emtree terms ('Ginkgo biloba', 'altitude disease'). The full search strategy for database is provided in the online supplementary file. References from retrieved articles were also examined to identify other relevant articles.

Studies were included in the systematic review if they (1) were randomised controlled trials (RCTs) of healthy non-acclimatised adult between the ages of 18 and 60 years; (2) compared GBE with placebo; (3) were conducted in humans; and (4) were studies that diagnosed AMS using the Lake Louise Score or the Environmental Symptom Questionnaire Acute Mountain Sickness-Cerebral score (AMS-C). We excluded studies with subjects who were pregnant and had symptoms consistent with AMS at baseline. Studies were also excluded if they were irrelevant to the aim of the study, were animal studies, lacked a placebo group, or were published as review articles, case reports, editorials or letters. The systematic review and meta-analysis was conducted under the Preferred Reporting Items for Systematic Reviews and Meta-Analyses guidelines (see online online supplementary checklist).

\section{Outcome measures}

AMS was defined as an AMS-C score $\geq 0.7$ or an LLS $\geq 3$ with headache. The primary outcome was the relative risk (RR) of AMS in participants receiving GBE for prophylaxis. We only extracted data when they were available in dichotomous form. The secondary outcomes of the included studies are summarised in online supplementary table 1 .

\section{Data extraction and assessment of methodological quality}

Two reviewers (T-YT and Y-CS) independently screened the titles and abstracts of all articles identified from the search strategy. Inter-reviewer disagreements concerning the inclusion or exclusion of a study were resolved by consensus and, if necessary, consultation with a third reviewer (S-HW).

The Cochrane Collaboration's tool was used to assess the risk of selection, performance, detection, attrition and reporting biases in the included randomised trials. ${ }^{21}$ We defined studies as 'high risk of bias' if one or more key domains is taken as high risk in the checklist. All coauthors discussed and made the final decisions about the overall risk of bias in the included trials. If data were not readily available or clear, we contacted the first authors and the corresponding authors to get further information. If studies were found to be at high risk of bias, meta-analyses stratified by study quality were performed.

Both reviewers independently extracted data from the articles selected for inclusion. The extracted data included the name of the first author, year of publication, numbers of participants, gender, starting and final altitudes, AMS scoring definitions, prescriptions of GBE, days of treatment prior to ascent, and number of individuals with AMS in the treatment and control groups.

\section{Data collection, data processing and primary data analysis}

Pooled RRs with corresponding 95\% CIs are derived for all studies and different subgroups of interest. The main outcome measure was the RR of AMS in participants receiving GBE for prophylaxis. Random-effect models with DerSimonian and Laird method were selected for these analyses. The pooled risk difference (RD) was also measured as the alternative outcome. The pooled RD is the difference between the observed risks (proportions of participants with AMS) in the two groups.

We conducted subgroup analyses based on the quality of studies, starting altitude, number of treatment days before ascending and dosage of GBE. ${ }^{22-24}$ Between-study heterogeneity was evaluated with the $\mathrm{I}^{2}$ statistic. ${ }^{25}$ The Egger regression asymmetry test and Begg adjusted rank correlation test were applied for assessment of potential publication bias. ${ }^{26}{ }^{27}$ We also conducted sensitivity analysis to evaluate the influence of each study on the overall pooled estimate. In dealing with zero cells, we add 0.5 to all cells of the $2 \times 2$ table for the study. Analyses were all conducted using STATA V.11.0. All statistical tests were two-sided and were considered significant when the $p$ value was 0.05 or less.

\section{Patient and public involvement statement}

Participants and the public sector were not directly involved in the design and conduct of this study. 


\section{0 reports identified from database search \\ (Cochrane Library, EMBASE, Google scholar, and PubMed databases)}

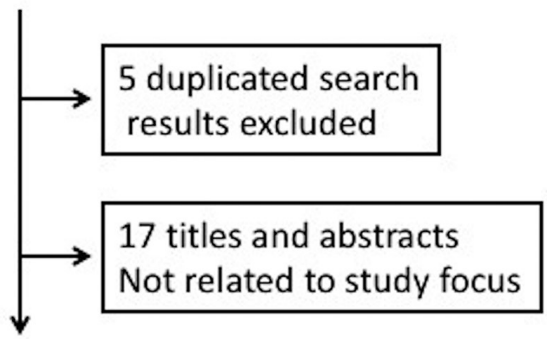

38 reports for further evaluation

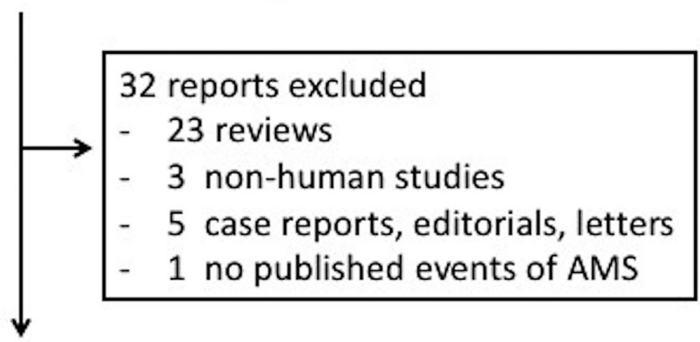

\section{6 reports included in the meta-analysis}

Figure 1 Trial selection algorithm. AMS, acute mountain sickness.

\section{RESULTS}

The literature search and study selection process are summarised in figure 1 . After the exclusion of duplicate studies, non-relevant studies and other studies that met the exclusion criteria based on a screening of article titles and abstracts, 38 potentially relevant studies were retrieved for full review.

One publication was retrieved by hand search of references. In this study, Wang et $a l^{28}$ compared the prophylactic effect of GBE with that of other Chinese medications on AMS. However, the study had no placebo group design ${ }^{29}$ and had to be excluded from our meta-analysis.

In the randomised, double-blind study by Ke et al in $2013,{ }^{20}$ AMS was reported as a secondary outcome and the number of events in each group was not reported. We contacted the first and corresponding authors by email but (as of 12 June 2018) received no response. Since the published data could not be included for analysis, we excluded this study.

Six published articles met all eligibility criteria after a careful review process. ${ }^{13}{ }^{15-19}$ In the article published by Leadbetter $e t a l,{ }^{19}$ two RCTs were conducted. As a result, a total of 7 study groups with 451 participants were enrolled. The characteristics of these studies and the participants are listed in table 1. Four study groups ${ }^{13151619}$ demonstrated the efficacy of GBE in preventing AMS, while three ${ }^{17-19}$ did not. All studies had small numbers of subjects except the one by Gertsch and colleagues. ${ }^{17}$

\begin{tabular}{|c|c|c|c|c|c|c|}
\hline & Participants (n) & Male (\%) & $\begin{array}{l}\text { Starting } \\
\text { altitude (m) }\end{array}$ & $\begin{array}{l}\text { Altitude } \\
\text { reached }(\mathrm{m})\end{array}$ & $\begin{array}{l}\text { Ascent rate } \\
\text { (m/hour) }\end{array}$ & AMS definition \\
\hline Gertsch et al, ${ }^{16} 2002$ & 26 & 46 & 0 & 4205 & 1402 & LLS $\geq 3$ with HA \\
\hline Gertsch et al, ${ }^{17} 2004$ & 243 & 70 & $4280-4358$ & 4928 & $10-20$ & LLS $\geq 3$ with HA \\
\hline $\begin{array}{l}\text { Leadbetter et al, }{ }^{19} \\
2009 \text { study } 1\end{array}$ & 40 & 45 & 2000 & 4300 & 1150 & AMS-C $\geq 0.7$ + LLS $\geq 3$ with $\mathrm{HA}$ \\
\hline $\begin{array}{l}\text { Leadbetter et al, }{ }^{19} \\
2009 \text { study } 2\end{array}$ & 37 & 44 & 2000 & 4300 & 1150 & AMS-C $\geq 0.7+L L S \geq 3$ with $\mathrm{HA}$ \\
\hline
\end{tabular}

AMS, acute mountain sickness; AMS-C, the Environmental Symptom Questionnaire III Acute Mountain Sickness-Cerebral score; HA, headache; LLS, Lake Louise Score. 
Table 2 Characteristics of included studies, sources, dosage and duration of ginkgo biloba

\begin{tabular}{|c|c|c|c|}
\hline & Ginkgo biloba extract source & Dose & $\begin{array}{l}\text { Days of treatment } \\
\text { prior to ascent }\end{array}$ \\
\hline Roncin et al,,$^{15} 1996$ & Tanakan DCl: EGb 761, Ipsen, Paris, France & $60 \mathrm{mg}$ twice daily & 0 \\
\hline Gertsch et al, ${ }^{16} 2002$ & GK501 Memfit, EGb 761, Pharmaton & $60 \mathrm{mg}$ three times a day & 1 \\
\hline Gertsch et al, ${ }^{17} 2004$ & GK501 International, Pharmaton & 120 mg twice daily & $1-2$ \\
\hline Chow et $a l,{ }^{18} 2005$ & Ginkgo biloba $120 \mathrm{mg}$, Vegetarian NOW Foods & 120 mg twice daily & 5 \\
\hline Moraga et $a l,{ }^{13} 2007$ & EGb 761 Rokan, Andromaco Laboratories, Chile & $80 \mathrm{mg}$ twice daily & 1 \\
\hline $\begin{array}{l}\text { Leadbetter et al, }{ }^{19} \\
2009, \text { study } 1\end{array}$ & Spectrum Quality, Laboratories Products & $120 \mathrm{mg}$ twice daily & 4 \\
\hline $\begin{array}{l}\text { Leadbetter et al, }{ }^{19} \\
2009, \text { study } 2\end{array}$ & Technical Sourcing & 120 mg twice daily & 3 \\
\hline
\end{tabular}

Of note, participants in the study conducted by Gertsch et $a l^{17}$ published in 2004 started GBE treatment at high altitude (4280-4358m), which was different from the other studies. Further information such as study dosage, prescription frequency, number of days prior to ascending and source of GBE is summarised in table 2. The number of AMS events and its incidence is summarised in figure 2. The quality of evidence of these studies as assessed by Cochrane Collaboration's tool is presented in table 3. Two of six articles were not double-blinded and both of them included male participants only. ${ }^{13} 15$ The study conducted by Gertsch et $a l^{16}$ in 2002 used 'first-come firstserved basis' after receiving signed consent. Therefore, we judge it as 'unclear random-sequence generation'. In addition, we appraised it as incomplete outcome data (attrition bias) because the study presented data on only 26 subjects when the intention was to enrol 100 subjects.

In the primary meta-analysis of all seven study groups, GBE showed trend of AMS prophylaxis, but it is not statistically significant $(\mathrm{RR}=0.68 ; 95 \% \mathrm{CI} 0.45$ to $1.04 ; \mathrm{p}=0.08)$ (figure 2). The $\mathrm{I}^{2}$ statistic was $58.7 \%(\mathrm{p}=0.02)$, indicating substantial heterogeneity. The pooled RD revealed a significant risk reduction in participants who use GBE ( $\mathrm{RD}=-25 \%$; 95\% CI, from a reduction of $45 \%$ to $6 \%$; $\mathrm{p}<0.001$ ) (figure 3). After excluding three high-risk bias studies, ${ }^{13}{ }^{15} 16$ the $\mathrm{I}^{2}$ statistic became $40.2 \%(\mathrm{p}=0.17)$ and the result did not change ( $\mathrm{RR}=0.84 ; 95 \%$ CI 0.59 to 1.21 ; $\mathrm{p}=0.36$ ). In the same subgroup the pooled $\mathrm{RD}$ is also not statistically significant $(\mathrm{RD}=-9.7 \%$; $95 \% \mathrm{CI}$, from a reduction of $27.4 \%$ to $7.9 \% ; \mathrm{p}=0.28)$. The Egger's test and Begg's test ( $\mathrm{p}=0.22$ and $\mathrm{p}=0.31$, respectively) indicate the absence of statistical evidence of publication bias after excluding our presumed high-risk bias articles.

Sensitivity analysis was conducted by removing one trial at a time to determine what influence each study had on the pooled analysis. The pooled result seemed to be robust. For example, removing the study conducted by Leadbetter et al in $2009^{19}$ only changed the pooled estimate from 0.68 to 0.74 (95\% CI 0.48 to 1.16 ; $p=0.19$; see online supplementary figure 1 ).

The results of several preplanned subgroup analyses were similar. Excluding the study by Gertsch and colleagues in $2004,{ }^{17}$ GBE was not prophylactic when the starting altitude was below $2500 \mathrm{~m} \quad(\mathrm{RR}=0.56$; 95\% CI 0.31 to 1.01$).{ }^{1315161819}$ Regarding the number of treatment days before ascending, GBE was not prophylactic when given ' $3-5$ days prior to ascent' 1819 ( $\mathrm{RR}=0.72 ; 95 \% \mathrm{CI} 0.41$ to 1.26 ) or ' $0-2$ days prior to ascent $^{13}{ }^{15-17}$ ( $\mathrm{RR}=0.56 ; 95 \%$ CI 0.25 to 1.25 ). Dosage of GBE was also not prophylactic for AMS when given 'less than $200 \mathrm{mg}$ per day' ${ }^{13} 1516$ ( $\mathrm{RR}=0.16$; 95\% CI 0.01 to 2.57$)$ or 'more than $200 \mathrm{mg}$ per day'17-19 $(\mathrm{RR}=0.84$;

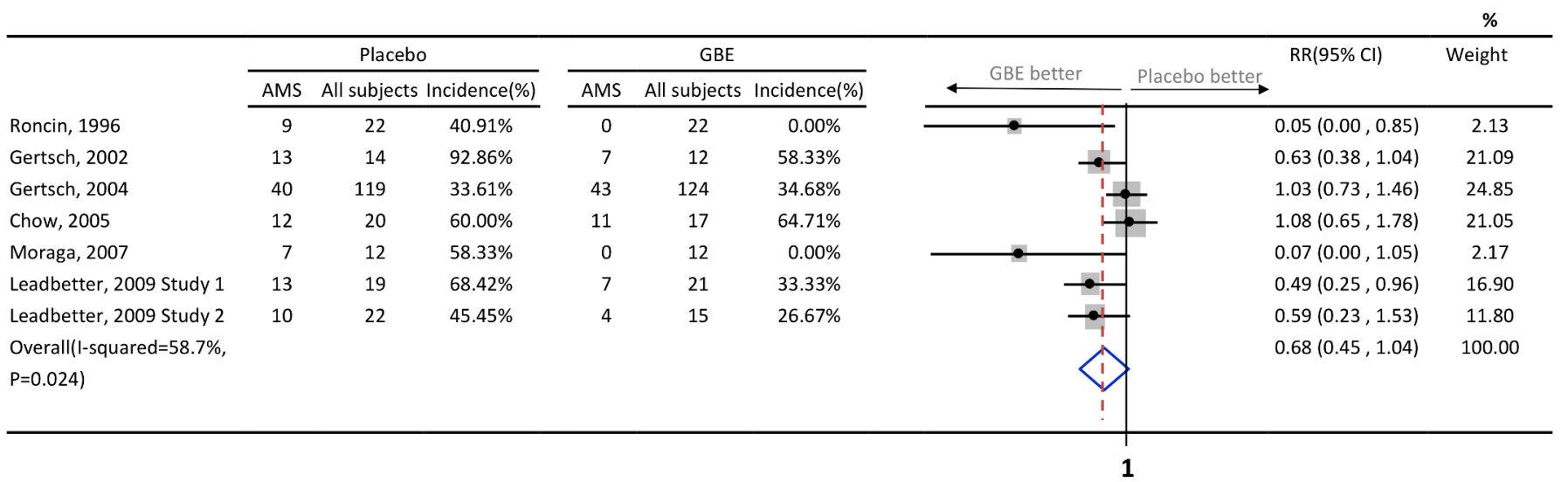

Figure 2 Events of acute mountain sickness between placebo and GBE, and forest plot of meta-analysis. AMS, acute mountain sickness; GBE, ginkgo biloba extract; RR, relative risk. 
Table 3 Risk of bias in included studies

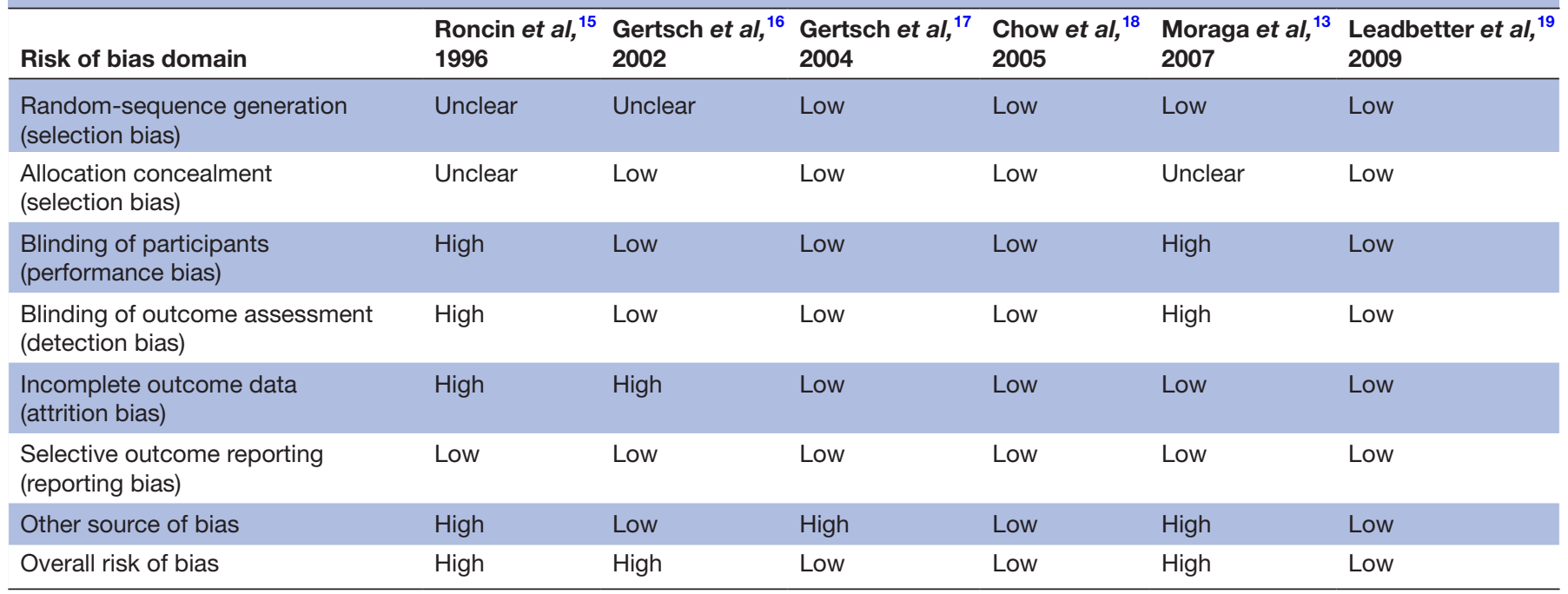

$95 \%$ CI 0.59 to 1.21$)$. Data on the number of participants and enrolled studies in each subgroup are summarised in online supplementary table 2.

\section{DISCUSSION}

To our knowledge, this is the first meta-analysis of RCTs evaluating GBE as an AMS prophylactic. In pooled analyses, we found that although GBE may tend towards AMS prophylaxis, it had no statistically significant prophylactic effect $(\mathrm{RR}=0.68 ; 95 \% \mathrm{CI} 0.45$ to $1.04 ; \mathrm{p}=0.08)$. The results of several subgroup analyses were similar. GBE also failed to show benefits in preventing AMS in low-risk bias studies, studies in which the starting altitude was low, studies differing in the initial treatment regimen prior to ascent and different dosage of GBE.

The effectiveness of GBE in AMS prophylaxis has been reported. $^{13151619}$ Zhang and colleagues ${ }^{29}$ in 2003 reported that GBE was the most effective of six Chinese medicines tested for AMS prophylaxis. GBE has been used primarily for the treatment of dementias (eg, Alzheimer's disease), peripheral vascular diseases (eg, intermittent claudication) and neurosensory problems (eg, tinnitus). ${ }^{30}$ Hypotheses have been proposed to explain the possible role that GBE plays in preventing AMS. Hypoxia is a common feature of AMS. Several studies have suggested that nitric oxide (NO) may play a pathogenic role in AMS by mediating hypoxia-induced cerebral vasodilation in humans. ${ }^{11-13}$ GBE was found to be an NO scavenger. NO scavenging can result in decreased intracellular NO level. ${ }^{14}$ Furthermore, GBE may inhibit phosphodiesterase activity, thus enhancing relaxation of parietal smooth muscle cells and so lead to vasodilation of parietal vessels. Vasodilation in turn increases tissue perfusion and decreases local hypoxia. ${ }^{14}$ Other potential mechanisms include increasing endogenous antioxidants, ${ }^{31}$ reducing free-radical production ${ }^{32}$ and reducing lung leak during hypoxia. ${ }^{33}$ GBE was also

\begin{tabular}{|c|c|c|c|}
\hline \multirow{2}{*}{$\begin{array}{l}\text { Study } \\
\text { ID }\end{array}$} & & \multicolumn{2}{|r|}{$\%$} \\
\hline & & $\mathrm{RD}(95 \% \mathrm{Cl})$ & Weight \\
\hline Roncin, 1996 & $\rightarrow$ & $-0.41(-0.62,-0.20)$ & 15.80 \\
\hline Gertsch, 2002 & 1 & $-0.35(-0.66,-0.04)$ & 13.00 \\
\hline Gertsch, 2004 & I & $0.01(-0.11,0.13)$ & 18.02 \\
\hline Chow, 2005 & 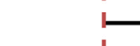 & $0.05(-0.27,0.36)$ & 12.93 \\
\hline Moraga, 2007 & $\rightarrow-1$ & $-0.58(-0.87,-0.30)$ & 13.60 \\
\hline Leadbetter, 2009 & & $-0.35(-0.64,-0.06)$ & 13.54 \\
\hline Leadbetter, 2009 & & $-0.19(-0.49,0.12)$ & 13.12 \\
\hline Overall(I-squared $=77.9 \%, P=0.000)$ & & $-0.25(-0.45,-0.06)$ & 100.00 \\
\hline NOTE:Weight are from random & I & & \\
\hline effects analysis & I & & \\
\hline
\end{tabular}

Figure 3 Pooled risk difference of enrolled studies. GBE, ginkgo biloba extract; RD, risk difference. 
shown to prevent high-altitude pulmonary oedema in a rat model. ${ }^{34}$

On the other hand, several studies failed to demonstrate the benefit of GBE in AMS prophylaxis. ${ }^{171820}$ The duration of therapy before ascent, dosage of GBE and differences in the altitude at which GBE is initiated may account for the conflicts between trial results. To test these hypotheses, we conducted subgroup analyses and obtained similar results to those obtained with the original pooled data. Another explanation for the differences in efficacy may be variation in the GBE composition. For instance, Leadbetter and colleagues ${ }^{19}$ in 2009 compared GBE from two different sources and found they differed in composition as well as ability to reduce the incidence and severity of AMS following rapid ascent to high altitude. The German Federal Institute for Drugs and Medicinal Devices Commission E recommends similar specifications for standardisation of GBE. All included studies used GBE that met the German E commission standard, but most of the studies use products from different companies. As an herbal supplement, more than $60 \%$ of GBE component is not mandated by law and composition may vary considerably between manufacturers. A lack of bioequivalence has been noted between brands of GBE. ${ }^{35} 36$

\section{Limitations}

Our systematic review has several limitations. First, to limit the influence of study biases on pooled evaluation, we decided to only include RCTs. However, there were few RCTs in this field. Moreover, only four of six RCTs were double-blinded. Second, because of the difficulty in carrying out high-altitude medicine studies, many studies involved only a small number of cases. In our primary pooled analysis, a total of 451 participants were enrolled. Insufficient power may be an issue in this meta-analysis. There are not enough data to show the statistically significant effect of GBE on preventing AMS, and further studies are warranted. Third, the participants were predominantly adult men, and whether there is gender or age difference between treatment (GBE vs placebo) groups or response (no AMS vs AMS) groups is unknown. Fourth, GBE is a complex mixture of natural components. It is difficult to standardise all components. A lack of consistency between commercially available GBE preparations may explain these differing results. Finally, differences between studies in factors such as the strength, rate of ascent and other characteristics of participants may also account for inconsistent results.

\section{CONCLUSION}

The currently available data suggest that although GBE may tend towards AMS prophylaxis, there are not enough data to show the statistically significant effect of GBE on preventing AMS. Further large randomised control studies are warranted.

Contributors T-YT analysed and interpreted the data and was a major contributor to writing the manuscript. S-HW interpreted the data. Y-KL supervised the study and interpreted the data. Y-CS interpreted the data and wrote the manuscript. All authors read and approved the final manuscript.

Funding The authors have not declared a specific grant for this research from any funding agency in the public, commercial or not-for-profit sectors.

Competing interests None declared.

Patient consent Not required.

Ethics approval The Institutional Review Board of Dalin Tzu Chi Hospital, Buddhist Tzu Chi Medical Foundation, Taiwan, approved the protocol.

Provenance and peer review Not commissioned; externally peer reviewed.

Data sharing statement Extra data can be accessed via the Dryad Data Repository at http://datadryad.org/ with the doi: 10.5061/dryad.35h13bg.

Open access This is an open access article distributed in accordance with the Creative Commons Attribution Non Commercial (CC BY-NC 4.0) license, which permits others to distribute, remix, adapt, build upon this work non-commercially, and license their derivative works on different terms, provided the original work is properly cited, appropriate credit is given, any changes made indicated, and the use is non-commercial. See: http://creativecommons.org/licenses/by-nc/4.0/.

\section{REFERENCES}

1. Honigman B, Theis MK, Koziol-McLain J, et al. Acute mountain sickness in a general tourist population at moderate altitudes. Ann Intern Med 1993;118:587-92.

2. Roach RC BP, Hackett PH, Oelz O. The Lake Louise acute mountain sickness scoring system. In: Sutton JR, Coates G, Huston CS, eds. Hypoxia and molecular medicine: proceedings of the 8th international hypoxia symposium. Lake Louise, Alberta, Canada. Burlington, VT: Queen City Printer, 1993:272-4.

3. Sampson JB, Cymerman A, Burse RL, et al. Procedures for the measurement of acute mountain sickness. Aviat Space Environ Med 1983;54(12 Pt 1):1063-73.

4. Basnyat B, Murdoch DR. High-altitude illness. Lancet 2003;361:1967-74.

5. Wang SH, Chen YC, Kao WF, et al. Epidemiology of acute mountain sickness on Jade Mountain, Taiwan: an annual prospective observational study. High Alt Med Biol 2010;11:43-9.

6. Chan CW, Lin YC, Chiu YH, et al. Incidence and risk factors associated with acute mountain sickness in children trekking on Jade Mountain, Taiwan. J Travel Med 2016;23:tav008.

7. Hackett PH, Yarnell PR, Hill R, et al. High-altitude cerebral edema evaluated with magnetic resonance imaging: clinical correlation and pathophysiology. JAMA 1998;280:1920-5.

8. Schoene RB. Illnesses at high altitude. Chest 2008;134:402-16.

9. Zafren K. Prevention of high altitude illness. Travel Med Infect Dis 2014;12:29-39.

10. Seupaul RA, Welch JL, Malka ST, et al. Pharmacologic prophylaxis for acute mountain sickness: a systematic shortcut review. Ann Emerg Med 2012;59:307-17.

11. Roach RC, Hackett PH. Frontiers of hypoxia research: acute mountain sickness. J Exp Biol 2001;204(Pt 18):3161-70.

12. Van Mil AH, Spilt A, Van Buchem MA, et al. Nitric oxide mediates hypoxia-induced cerebral vasodilation in humans. J Appl Physiol 2002;92:962-6.

13. Moraga FA, Flores A, Serra J, et al. Ginkgo biloba decreases acute mountain sickness in people ascending to high altitude at Ollagüe (3696 m) in northern Chile. Wilderness Environ Med 2007;18:251-7.

14. Marcocci L, Maguire JJ, Droy-Lefaix MT, et al. The nitric oxidescavenging properties of Ginkgo biloba extract EGb 761. Biochem Biophys Res Commun 1994;201:748-55.

15. Roncin JP, Schwartz F, D'Arbigny P. EGb 761 in control of acute mountain sickness and vascular reactivity to cold exposure. Aviat Space Environ Med 1996;67:445-52.

16. Gertsch JH, Seto TB, Mor J, et al. Ginkgo biloba for the prevention of severe acute mountain sickness (AMS) starting one day before rapid ascent. High Alt Med Biol 2002;3:29-37.

17. Gertsch JH, Basnyat B, Johnson EW, et al. Randomised, double blind, placebo controlled comparison of ginkgo biloba and acetazolamide for prevention of acute mountain sickness among Himalayan trekkers: the prevention of high altitude illness trial (PHAIT). BMJ 2004;328:797.

18. Chow T, Browne V, Heileson HL, et al. Ginkgo biloba and acetazolamide prophylaxis for acute mountain sickness: a randomized, placebo-controlled trial. Arch Intern Med 2005;165:296-301. 
19 Leadbetter G, Keyes LE, Maakestad KM, et al. Ginkgo biloba doesand does not-prevent acute mountain sickness. Wilderness Environ Med 2009;20:66-71.

20. Ke T, Wang J, Swenson ER, et al. Effect of acetazolamide and gingko biloba on the human pulmonary vascular response to an acute altitude ascent. High Alt Med Biol 2013;14:162-7.

21. Higgins JP, Altman DG, Gøtzsche PC, et al. The cochrane collaboration's tool for assessing risk of bias in randomised trials. BMJ 2011;343:d5928.

22. Hackett $\mathrm{PH}$, Roach RC, Illness H-ANew England Journal of Medicine 2001;345:107-14.

23. van Patot MC, Keyes LE, Leadbetter G, et al. Ginkgo biloba for prevention of acute mountain sickness: does it work? High Alt Med Biol 2009;10:33-43.

24. Dumont L, Mardirosoff $C$, Tramèr MR. Efficacy and harm of pharmacological prevention of acute mountain sickness: quantitative systematic review. BMJ 2000;321:267-72.

25. Higgins JP, Thompson SG, Deeks JJ, et al. Measuring inconsistency in meta-analyses. BMJ 2003;327:557-60.

26. Begg CB, Mazumdar M. Operating characteristics of a rank correlation test for publication bias. Biometrics 1994;50:1088-101.

27. Egger M, Davey Smith G, Schneider M, et al. Bias in meta-analysis detected by a simple, graphical test. BMJ 1997;315:629-34.
28. Wang J, Xiong X, Xing Y, et al. Chinese herbal medicine for acute mountain sickness: a systematic review of randomized controlled trials. Evid Based Complement Alternat Med 2013;2013:1-8.

29. Zhang HJY XZ, Ha ZD. Role of six different medicines in the symptomatic scores of benign form of acute mountain sickness. Medical Journal of National Defending Forces in Northwest China 2003;24:341-3.

30. Sierpina VS, Wollschlaeger B, Blumenthal M. Ginkgo biloba. Am Fam Physician 2003;68:923-6.

31. Louaji A, Harraga S, Godot V, et al. The effect of ginkgo biloba extract on free radical production in hypoxic rats. Biol Pharm Bull 2001;24:710-2.

32. Naik SR, Pilgaonkar VW, Panda VS. Evaluation of antioxidant activity of Ginkgo biloba phytosomes in rat brain. Phytother Res 2006;20:1013-6.

33. Liu KX, Wu WK, He W, et al. Ginkgo biloba extract (EGb 761) attenuates lung injury induced by intestinal ischemia/reperfusion in rats: roles of oxidative stress and nitric oxide. World J Gastroenterol 2007;13:299-305.

34. Berg JT. Ginkgo biloba extract prevents high altitude pulmonary edema in rats. High Alt Med Biol 2004;5:429-34.

35. De Smet PA. Herbal remedies. N Engl J Med 2002;347:2046-56.

36. Kressmann S, Müller WE, Blume HH. Pharmaceutical quality of different Ginkgo biloba brands. J Pharm Pharmacol 2002;54:661-9. 